\title{
Asian variant of intravascular large B-cell lymphoma: a comparison of clinical features based on involvement of the central nervous system
}

\author{
Sang Eun Yoon ${ }^{1}$, Won Seog Kim ${ }^{1}$, and Seok Jin Kim ${ }^{1,2}$
}

${ }^{1}$ Division of Hematology-Oncology, Department of Medicine, Samsung Medical Center, Sungkyunkwan University School of Medicine, Seoul; ${ }^{2}$ Department of Health Sciences and Technology, Samsung Advanced Institute for Health Sciences and Technology, Sungkyunkwan University, Seoul, Korea

Received: November 5, 2018 Revised : November 12, 2018 Accepted: November 17, 2018

\section{Correspondence to}

Seok Jin Kim, M.D.

Division of Hematology-

Oncology, Department of

Medicine, Samsung Medical

Center, Sungkyunkwan

University School of Medicine,

81 Irwon-ro, Gangnam-gu, Seoul 06351, Korea

Tel: +82-2-3410-1766

Fax: +82-2-3410-1754

E-mail: kstwoh@skku.edu
Background/Aims: There are limited data about the influence of the central nervous system (CNS) involvement on the prognosis for patients with the Asian variant of intravascular large B-cell lymphoma (IVLBCL).

Methods: We analyzed 46 patients who were diagnosed with IVLBCL between 2001 and 2018. All patients were treated with rituximab, cyclophosphamide, doxorubicin, vincristine, and prednisone (R-CHOP).

Results: CNS involvement was diagnosed by cerebrospinal fluid analysis $(\mathrm{n}=6$ ) and brain imaging $(\mathrm{n}=5)$ in 11 patients at diagnosis, and four cases with CNS relapse were found. Thus, 15 patients had CNS involvement (15/46, 33\%). The clinical characteristics were not different between patients with and without CNS involvement, but all patients with CNS involvement belonged to the high-risk group of CNS-International Prognostic Index (IPI). Thirty-one patients achieved a complete response $(67 \%, 31 / 46)$ whereas eight patients showed disease progression and six patients died after the first or second cycle of R-CHOP. CNS-directed therapy such as high-dose methotrexate was combined with R-CHOP for patients with CNS involvement, and five patients were alive without relapse. The median overall survival of all patients was 45.0 months, and overall survival was not different according to the involvement of CNS.

Conclusions: The treatment outcome of patients with the Asian variant of IVLB$\mathrm{CL}$ is still not satisfactory. The prediction of CNS involvement based on the clinical features including CNS-IPI score might not serve to identify patients at high risk of CNS involvement, either. Thus, more effective strategies for diagnosis and treatment should be developed.

Keywords: Lymphoma, large B-cell, diffuse; Intravascular lymphoma; Central nervous system

\section{INTRODUCTION}

Intravascular large B-cell lymphoma (IVLBCL) is a rare form of large B-cell lymphoma with pathological findings of intravascular proliferation and/or sinusoidal involvement of lymphoma cells [1]. IVLBCL is classified as a subtype of extranodal large B-cell lymphoma in the World Health Organization (WHO) classification because of its distinctive clinical features [2]. Although the recently updated WHO classification suggested classic, cutaneous, and hemophagocytic syndrome-associated variants according to clinical and laboratory features, 
Asian and Western variants according to their geographic distribution were still commonly used $[3,4]$. Compared with the Western variant, where skin involvement was common, the Asian variant was reported to involve more frequently the liver, spleen, and bone marrow [5]. A hemophagocytic syndrome-associated variant could be referred to as the Asian variant because hemophagocytic lymphohistiocytosis (HLH) was more common in Asian patients with IVLBCL, whereas HLH is a rare event in those with the Western variant [6]. A population-based study of IVLBCL in the United States reported that the incidence of IVLBCL increased significantly from 2000 to 2013 [7]. This might be associated with better understanding of the clinical and laboratory features of this disease entity. Nevertheless, the diagnosis of IVLBL remains problematic, and it is still difficult to diagnose IVLBCL because of the lack of overt lymphadenopathy and peripheral blood involvement. In particular, the Asian variant might be commonly misdiagnosed as other disorders such as infections because of its common association with HLH. Thus, timely diagnosis and immediate treatment remain as a challenge to improve outcomes for patients with the Asian variant.

Given that the Asian variant more frequently presents as stage IV disease involving the liver and bone marrow as well as HLH, the outcome of affected patients might be inferior to that of those with diffuse large B-cell lymphoma, not otherwise specified (DLBCL-NOS). Furthermore, there are limited data regarding central nervous system (CNS) involvement in the Asian variant because this is relatively more frequent in the Western variant [5]. The negative impact of CNS involvement on the prognosis of DLBCL-NOS was well known even in the era of rituximab [8-11]. However, there is still no consensus on the treatment approach for CNS involvement in patients with lymphomas including IVLBCL. Therefore, we analyzed the clinical features and treatment outcomes of patients with the Asian variant of IVLBCL and also compared their survival outcomes based on CNS involvement to evaluate its impact on the prognosis.

\section{METHODS}

\section{Patients}

We reviewed the electronic medical records of patients who were diagnosed with IVLBCL at Samsung Medical Center between 2001 and 2018. The diagnosis was based on histopathological criteria including the presence of CD2o-positive neoplastic B cells with large cell morphology and intravascular or sinusoidal proliferation of lymphoma cells. All patients were evaluated for staging work-ups in clinical practice including physical examinations, complete blood cell counts, serum biochemistry, computed tomography scans of chest and abdominal pelvis, bone marrow aspiration and biopsy, and ${ }^{18} \mathrm{~F}$-fluorodeoxyglucose positron emission tomography/ computed tomography scans. Additional work-up procedures, such as brain or spine magnetic resonance imaging (MRI), cerebrospinal fluid (CSF) analysis, and cytological examination of pleural fluid, were performed if indicated clinically. Stage was determined using the Ann Arbor staging system, and the risk of prognosis was defined by the International Prognostic Index (IPI) consisting of age, Eastern Cooperative Oncology Group performance status, Ann Arbor stage, numbers of extranodal involvements, and serum lactate dehydrogenase (LDH) levels at diagnosis. This study was approved by the Institutional Review Board of Samsung Medical Center, Seoul, Korea, and the requirement for informed consent was waived because of the retrospective nature of the study (IRB File No: 2018-10-065).

\section{Data collection}

After reviewing the medical records, clinical features at the time of diagnosis were collected. CNS involvement was defined as the presence of a lymphoma in the brain parenchyma and/or spinal cord as demonstrated by MRI, or leptomeningeal involvement diagnosed by CSF analysis at the time of diagnosis or during or after treatment. As a predictive model for prognosis as well as CNS involvement, we gathered the data required for CNS-IPI and National Comprehensive Cancer Network (NCCN)-IPI because they showed better prognostic power compared with existing standard IPI [12,13]. As most patients were treated with R-CHOP chemotherapy regimens (rituximab $375 \mathrm{mg} / \mathrm{m}^{2}$ on day 1, cyclophosphamide $750 \mathrm{mg} / \mathrm{m}^{2}$, doxorubicin $50 \mathrm{mg} / \mathrm{m}^{2}$, vincristine $1.4 \mathrm{mg} / \mathrm{m}^{2}$ [maximum $2 \mathrm{mg}$ ] on day 1 , and prednisone $100 \mathrm{mg}$ on day 1 to 5 ), data including the number of cycles and dose intensity (cumulative dose in $\mathrm{mg}$ ) / (treatment duration in weeks) of R-CHOP were gathered. For patients with CNS in- 
volvement, CNS-directed therapy such as intrathecal (IT) or intravenous (IV) methotrexate (MTX) was added to R-CHOP in clinical practice. The IT chemotherapy regimens were MTX-based combination regimens (MTX $15 \mathrm{mg}$, Ara-C $30 \mathrm{mg} / \mathrm{m}^{2}$, hydrocortisone $15 \mathrm{mg} / \mathrm{m}^{2}$ ) or MTX single therapy (MTX $15 \mathrm{mg}$ IT), whereas the IV MTX regimen used 1 to $1.5 \mathrm{~g} / \mathrm{m}^{2}$ over 6 hours. These data about CNS-directed therapy were also collected. The treatment response to R-CHOP was re-determined by the Revised Response Criteria for Malignant Lymphoma [14]. High-dose chemotherapy followed by autologous stem cell transplantation (ASCT) was done in selected patients after the completion of R-CHOP chemotherapy (upfront ASCT) or salvage chemotherapy (salvage ASCT) according to the physicians' discretions. As this was a retrospective study, adverse events and toxicities were based on a review of medical records. According to the National Cancer Institute Common Terminology Criteria for Adverse Events (NCI-CTCAE) version 4.o, grade 3 or 4 adverse events were recorded.

\section{Statistical analysis}

Descriptive statistics are reported as proportions and medians. Intergroup comparisons were performed using Fisher's exact test for categorical variables and the Mann-Whitney nonparametric $U$ test for age. Progression-free survival (PFS) was calculated from the date of diagnosis to the first day of disease progression, relapse, death as a result of any cause, or last date of follow-up. Overall survival (OS) was calculated from the date of diagnosis to death or to the last date of follow-up. Survival curves were generated using the Kaplan-Meier method, and survival rates were compared using the log-rank test. Univariate and multivariate analysis was performed using Cox regression analysis. Two-sided $p$ values $<0.05$ were considered significant, and statistical analyses were performed using the statistical software package IBM PASW version 18.0 (SPSS Inc., Chicago, IL, USA).

\section{Ethical approval}

All procedures performed in studies involving human participants were in accordance with the ethical standards of the institutional and/or national research committee and with the 1964 Helsinki declaration and its later amendments or comparable ethical standards.

\section{RESULTS}

\section{Characteristics of patients at diagnosis}

Forty-six patients were diagnosed with IVLBCL, and their median age at diagnosis was 62 years (range, 34 to 82 ). Male patients predominated $(n=26,57 \%)$, and B symptoms were present in 31 patients (78\%) (Table 1 ). Hepatomegaly and/or splenomegaly were observed in 26 patients (57\%), whereas lymphadenopathy was less common $(\mathrm{n}=22,48 \%)$. Cytopenia affecting the hemoglobin level $(<11 \mathrm{~g} / \mathrm{dL})$ and/or platelet count $\left(<100 \times 10^{9} / \mathrm{L}\right)$ were also found frequently (Table 1$)$. The clinically presenting symptoms were diverse; however, fever was the most common symptom (27/46, 59\%) (Fig. 1A). Neurological symptoms, such as diplopia and facial palsy, and altered mental functions were the second most common symptoms $(15 / 46,33 \%)$. Bone marrow and liver were the most commonly involved extranodal organs ( $54 \%$ and $48 \%$, respectively) and were the most common sites of biopsy for diagnosis in this study (Fig. 1B). Accordingly, most patients were at stage IV disease $(n=42,91 \%)$ (Table 1 ). All patients had increased levels of serum LDH, and 30 patients had extremely high levels (more than three times the normal range) (Table 1 and Fig. 1 C). Sixteen patients (35\%) had HLH, and four patients showed severe hypercalcemia (up to $1.35 \mathrm{mmol} / \mathrm{L}$ ). Based on the IPI score, 44 patients (96\%) were classed as high-to-intermediate risk or high risk, whereas 44 patients (96\%) were classed as having a high-to-intermediate risk or a high risk by NCCN-IPI (Table 1). Classification according to the CNS-IPI score showed that 38 patients belonged to the high risk group, while only eight patients were in the low or intermediate risk categories (Table 1).

\section{Treatment outcomes}

All patients received R-CHOP as a first-line treatment after diagnosis with a median number of six cycles (range one to eight). At the end of treatment, 31 patients achieved a complete response (CR), whereas eight patients showed disease progression (Fig. 2). Six patients died after the first or second cycle of R-CHOP, and the causes of death were treatment-related adverse events including cytopenia, infectious complications, and pulmonary hemorrhage. One patient was lost to follow-up after the completion of the first cycle. Therefore, the CR rate based on intent to treat was $67 \%$ (31/46). According 
Table 1. Characteristics of the patients $(n=46)$

\begin{tabular}{|c|c|}
\hline Characteristic & No. (\%) \\
\hline \multicolumn{2}{|l|}{ Sex } \\
\hline Male & $26(57)$ \\
\hline Female & $20(43)$ \\
\hline \multicolumn{2}{|l|}{ Age, yr } \\
\hline$\leq 60$ & $20(44)$ \\
\hline$>60$ to $<75$ & $18(39)$ \\
\hline$\geq 75$ & $8(17)$ \\
\hline \multicolumn{2}{|c|}{ Eastern Cooperative Oncology Group } \\
\hline $0 / 1$ & $10(22)$ \\
\hline$\geq 2$ & $36(78)$ \\
\hline \multicolumn{2}{|l|}{ Serum LDH } \\
\hline Normal & o \\
\hline Increased $>1$ to $\leq 3$ & $16(35)$ \\
\hline Increased $>3$ & $30(65)$ \\
\hline \multicolumn{2}{|l|}{ B symptoms } \\
\hline Absence & $15(33)$ \\
\hline Presence & $31(67)$ \\
\hline \multicolumn{2}{|l|}{ Stage } \\
\hline I/II & $1 / 2(7)$ \\
\hline III/IV & $1 / 42(93)$ \\
\hline \multicolumn{2}{|l|}{ Bone marrow involvement } \\
\hline Absence & $21(46)$ \\
\hline Presence & $25(54)$ \\
\hline \multicolumn{2}{|c|}{ No. of extranodal involvement } \\
\hline $0 / 1$ & $4 / 11(33)$ \\
\hline$\geq 2$ & $31(67)$ \\
\hline \multicolumn{2}{|c|}{ Hepatomegaly and/or splenomegaly } \\
\hline Absence & $20(43)$ \\
\hline Presence & $26(57)$ \\
\hline \multicolumn{2}{|l|}{ Hemoglobin < $11 \mathrm{~g} / \mathrm{dL}$} \\
\hline Absence & $18(39)$ \\
\hline Presence & $28(61)$ \\
\hline \multicolumn{2}{|c|}{ Platelet count $<100 \times 10^{9} / \mathrm{L}$} \\
\hline Absence & $26(57)$ \\
\hline Presence & $20(43)$ \\
\hline \multicolumn{2}{|l|}{ Lymphadenopathy } \\
\hline Absence & $24(52)$ \\
\hline Presence & $22(48)$ \\
\hline \multicolumn{2}{|l|}{ Serum albumin } \\
\hline Normal & $19(41)$ \\
\hline Decreased $(<3.5 \mathrm{~g} / \mathrm{dL})$ & $27(59)$ \\
\hline \multicolumn{2}{|l|}{ IPI } \\
\hline L/LI & $2(4)$ \\
\hline $\mathrm{HI} / \mathrm{H}$ & $44(96)$ \\
\hline \multicolumn{2}{|l|}{ NCCN-IPI } \\
\hline $\mathrm{L} / \mathrm{LI}$ & $2(4)$ \\
\hline
\end{tabular}

Table 1. Continued

\begin{tabular}{lc}
\hline Characteristic & No. (\%) \\
\hline HI/H & $44(96)$ \\
CNS-IPI & 0 \\
Low & $8(17)$ \\
Intermediate & $38(83)$ \\
High & \\
Hemophagocytic lymphohistiocytosis & $30(65)$ \\
Absence & $16(35)$ \\
\hline Presence
\end{tabular}

$\mathrm{LDH}$, lactate dehydrogenase; IPI, International Prognostic Index; L/LI, low/low-intermediate; $\mathrm{HI} / \mathrm{H}$, high-intermediate/high; NCCN, National Comprehensive Cancer Network; CNS, central nervous system.

to physicians' decisions, the dosage of R-CHOP was reduced in 13 patients for elderly or frail patients. The administration of rituximab in the first cycle was delayed in 21 patients when considering the risk of infusion-related reaction and rituximab-related severe adverse events. As a result, the median dose-intensity of each drug was as follows: rituximab $200 \mathrm{mg} / \mathrm{wk}$ (range, 163 to 240), cyclophosphamide $393 \mathrm{mg} / \mathrm{wk}$ (range, 299 to 477), doxorubicin $26 \mathrm{mg} / \mathrm{wk}$ (range, 18 to 32), and vincristine $1 \mathrm{mg} / \mathrm{wk}$. The most frequently attenuated drugs were doxorubicin and vincristine, which were reduced by approximately $20 \%$ to $25 \%$. However, the overall response rate was not significantly different, regardless of the dose reduction or intensity (data not shown). The most common grade 3 or 4 toxicity seen was neutropenia or febrile neutropenia $(n=26,57 \%)$. Three patients developed pulmonary hemorrhage during the first cycle of R-CHOP, especially immediately after the infusion of rituximab. Two of them recovered; however, one patient died from massive pulmonary hemorrhage and subsequent infectious complications. Infusion-related reactions associated with rituximab such as fever, chills, and dyspnea were also observed in seven patients. Upfront ASCT was done for two patients including one patient with CNS involvement at diagnosis, and these patients were still alive at the time of analysis without evidence of relapse (Fig. 3). On the other hand, the outcome of six patients undergoing salvage ASCT after relapse was poor; thus, only one patient was alive. Likewise, patients with disease progression at the end of treatment with 

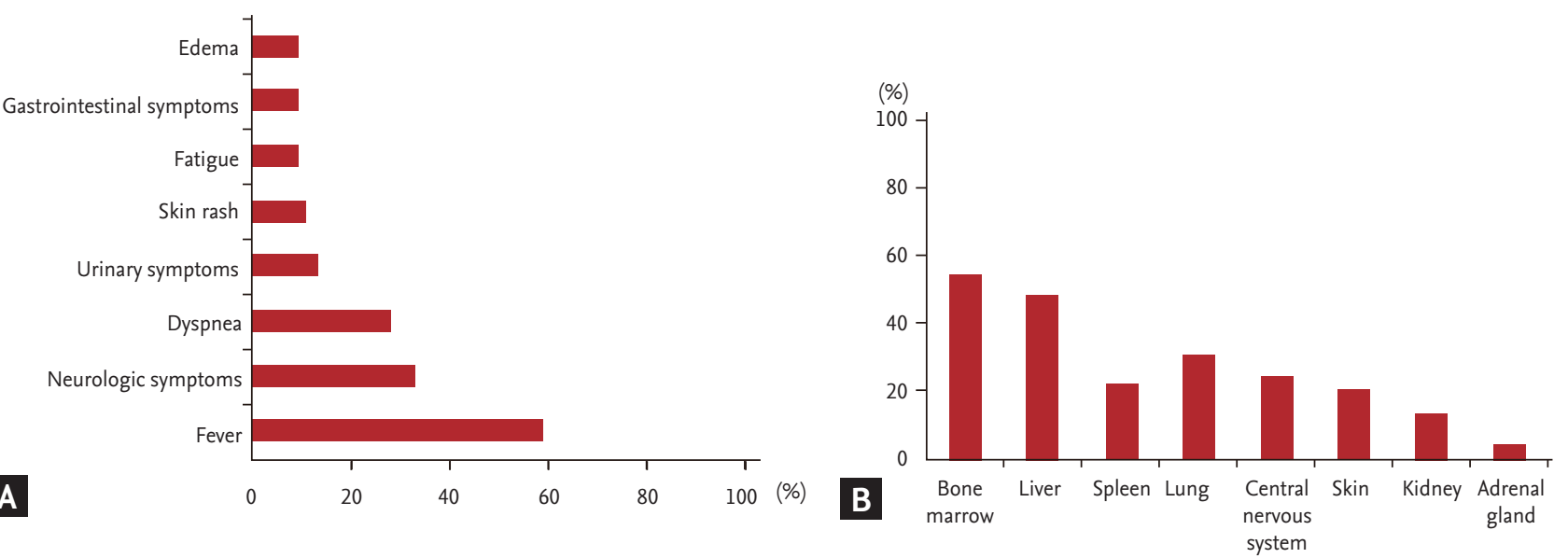

C
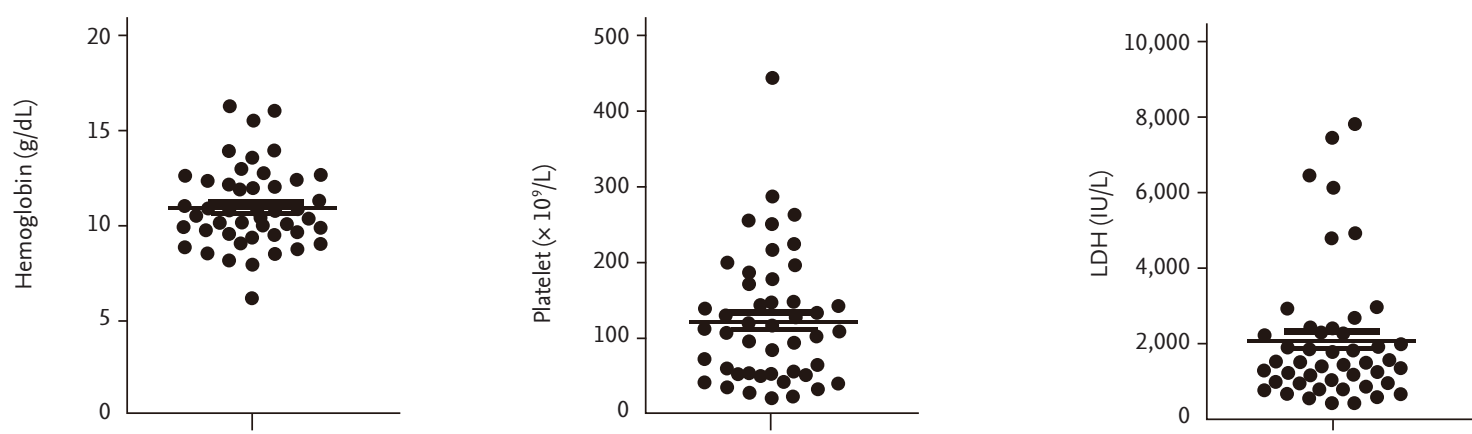

Figure 1. (A) Clinically presenting symptoms of the 46 patients. (B) The order of frequently involved organs. (C) Hemoglobin level $(\mathrm{g} / \mathrm{dL})$, platelet count $\left(\times 10^{6} / \mathrm{L}\right)$, and serum lactate dehydrogenase $(\mathrm{LDH})$ level $(\mathrm{IU} / \mathrm{L})$ at diagnosis.

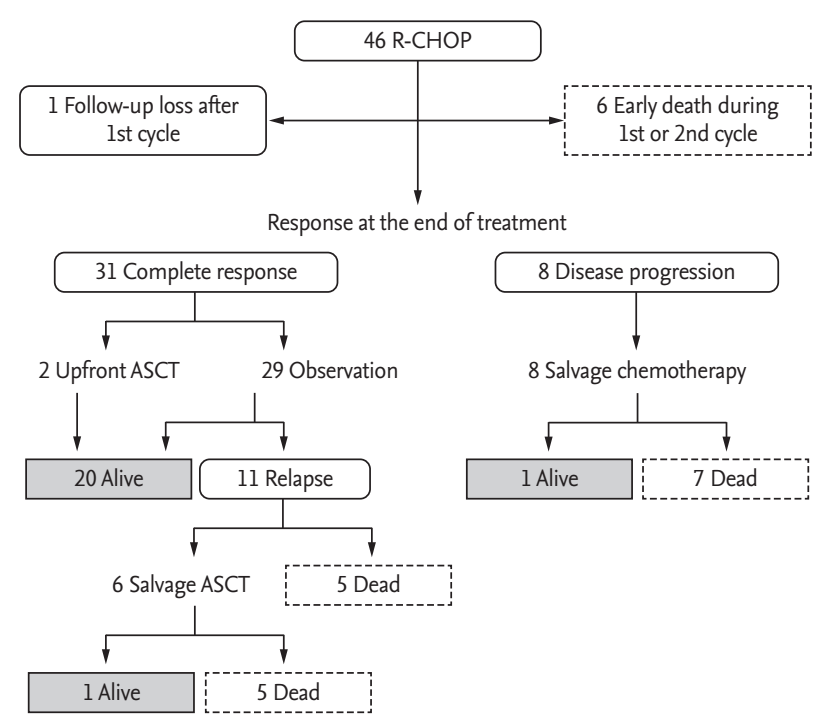

Figure 2. Treatment outcomes for the 46 patients. R-CHOP, rituximab, cyclophosphamide, doxorubicin, vincristine, and prednisone; ASCT, autologous stem cell transplantation.
R-CHOP showed dismal prognoses even after salvage chemotherapy except for one (Fig. 2).

\section{CNS involvement and treatment outcomes}

At diagnosis, 11 patients had CNS involvement (24\%) (Fig. 1B), and neurologic signs and symptoms were present in eight of them (8/11; 73\%). Neurologic symptoms were heterogeneous, including diplopia, headache, visual defect, hearing difficulty, facial palsy, and mental changes. The diagnosis of CNS involvement was done by CSF analysis via lumbar puncture $(n=6)$, while the remaining five patients were diagnosed by radiologic imaging such as MRI. Four patients developed CNS involvement during or after treatment with R-CHOP. As a result, 15 of the 46 patients had CNS involvement (33\%). A comparison of characteristics at diagnosis between patients with and without CNS involvement did not show significant differences (Table 2). However, all patients with CNS involvement belonged to the high-risk group according to CNS-IPI, whereas $74 \%$ of patients 


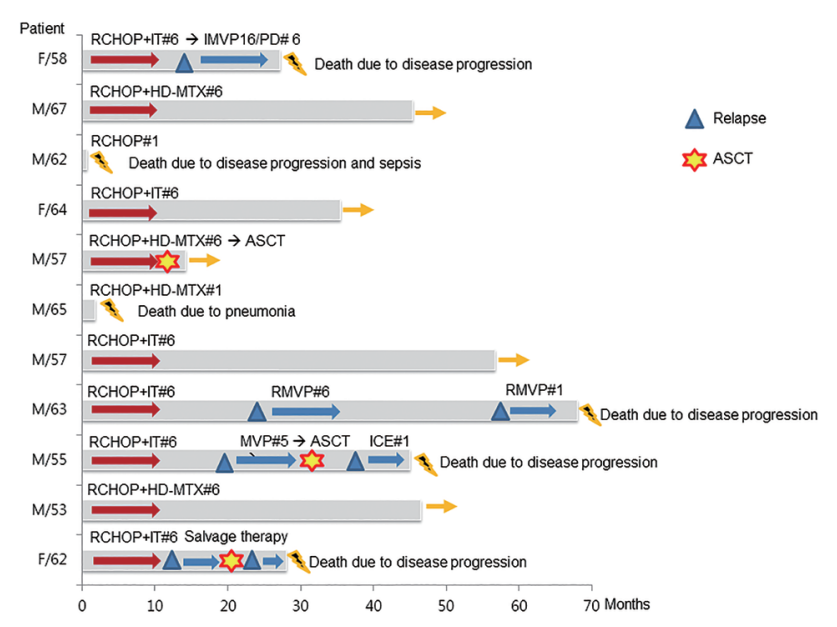

Figure 3. Clinical course and outcome of 11 patients with central nervous system (CNS) involvement at diagnosis. R-CHOP, rituximab, cyclophosphamide, doxorubicin, vincristine and prednisone; IT, intrathecal chemotherapy; HDMTX, high-dose methotrexate; IMVP16/PD, ifosfamide, methotrexate, etoposide and prednisone; ASCT, autologous stem cell transplantation; RMVP, rituximab, methotrexate, vincristine and procarbazine; ICE, ifosfamide, carboplatin and etoposide; \#, number of cycles.

without CNS involvement were grouped as high risk by CNS-IPI $(p=0.04)$ (Table 2). CNS-directed therapy was combined with R-CHOP in 11 patients who had CNS involvement at diagnosis. Among these patients, five patients were alive without evidence of relapse. However, six patients died because of disease relapse or early mortality caused by infectious complications. In particular, one patient relapsed after CR, and all patients died from disease progression even after ASCT (Fig. 3). Likewise, the outcomes of four patients who had CNS relapse after R-CHOP chemotherapy were poor. Three of these patients died from disease progression, and only one patient was alive after being salvaged successfully by CNS-directed chemotherapy.

\section{Survival analysis}

At a median follow-up of 47.0 months (95\% confidence interval [CI], 25.0 to 69.0), the median OS was 45.0 months (95\% CI, 25.8 to 64.2) (Fig. 4A). The median PFS was 23.0 months (95\% CI, 4.5 to 41.5 ), and the OS was significantly worse in patients with relapse or disease progression (Fig. 4B). The presence of thrombocytopenia at diagnosis had a significant association with OS $(p=$
0.010) (Fig. 4C). As most patients (93\%) had stage IV disease and belonged to the high or high-to-intermediate risk group according to the IPI and NCCN-IPI grades, these parameters could not discriminate patients with better and worse survival outcomes (data not shown). The CNS-IPI grading also failed to show a significant association with survival because 38 patients (83\%) were grouped as having high risk CNS-IPI (Table 1). Thus, the univariate analysis for OS using each parameter showed that only the presence of thrombocytopenia or anemia was significantly associated with a poor OS (Table 3). The presence of HLH and CNS involvement at diagnosis were not significantly associated with poor survival outcomes (Table 3 and Fig. 4D). However, none of these parameters showed independent associations with overall survival in the multivariate analysis (Table 3).

\section{DISCUSSION}

In our study, clinical features were consistent with criteria for the diagnosis of the Asian variant of IVLBCL including cytopenia, hepatosplenomegaly, and histopathological features [15]. The most common presenting symptoms were fever, neurologic symptoms, and dyspnea (Fig. 1A). All patients showed elevated levels of serum LDH, and more than $60 \%$ of them showed extremely elevated levels (Fig. 1C). Anemia and thrombocytopenia were also common and might be associated with HLH and hepatosplenomegaly (Table 1). Therefore, if Asian patients with fever or neurologic symptoms show highly elevated levels of serum LDH and cytopenia, the possibility of IVLBCL should be considered.

Early studies in Japan evaluating patients with IVLBCL by immunohistochemistry showed that more than $75 \%$ of IVLBCL cases were the activated B-cell-like $(\mathrm{ABC})$ type, and $\mathrm{CD}_{5}$, an unfavorable marker, was more frequently expressed in patients with IVLBCL than in those with DLBCL-NOS $[1,3]$. A recent next-generation sequencing of 25 cases with IVLBCL also reported that $44 \%$ had MYDS8 ${ }^{L 256 P}$ and $26 \%$ had CD 96 mutations, known to be associated with poor prognosis [16]. This double mutation was also identified by a recent study with exome sequencing of DLBCL biopsy samples, and its presence was significantly associated with poor prognosis, especially in the ABC type of DLBCL [17]. 
Table 2. Comparison of patients with and without CNS involvement

\begin{tabular}{|c|c|c|c|}
\hline \multirow{2}{*}{ Characteristic } & \multicolumn{2}{|c|}{ CNS involvement } & \multirow{2}{*}{$p$ value } \\
\hline & Presence $(n=15)$ & Absence $(n=31)$ & \\
\hline Sex & & & 0.128 \\
\hline Male & $11(73)$ & $15(48)$ & \\
\hline Female & $4(27)$ & $16(52)$ & \\
\hline Age, yr & & & 0.341 \\
\hline$\leq 60$ & $8(53)$ & $11(36)$ & \\
\hline$>60$ & $7(47)$ & $20(64)$ & \\
\hline ECOG & & & 0.999 \\
\hline o/1 & $3(20)$ & $7(23)$ & \\
\hline$\geq 2$ & $12(80)$ & $24(77)$ & \\
\hline Serum LDH & & & 0.520 \\
\hline Increased $\leq 3$ & $4(27)$ & $12(39)$ & \\
\hline Increased $>3$ & $11(73)$ & $19(61)$ & \\
\hline B symptoms & & & 0.514 \\
\hline Absence & $6(40)$ & $9(29)$ & \\
\hline Presence & $9(60)$ & $22(71)$ & \\
\hline Stage & & & 0.541 \\
\hline I/II & 0 & $3(10)$ & \\
\hline III/IV & $15(100)$ & $28(90)$ & \\
\hline Bone marrow involvement & & & 0.999 \\
\hline Absence & $7(47)$ & $14(45)$ & \\
\hline Presence & $8(53)$ & $17(55)$ & \\
\hline No. of extranodal involvement & & & 0.317 \\
\hline $0 / 1$ & $3(20)$ & $12(39)$ & \\
\hline$\geq 2$ & $12(80)$ & $19(61)$ & \\
\hline Hepatosplenomegaly & & & 0.204 \\
\hline Absence & $9(60)$ & $11(36)$ & \\
\hline Presence & $6(40)$ & $20(64)$ & \\
\hline Anemia $^{\mathrm{a}}$ and/or thrombocytopenia ${ }^{\mathrm{a}}$ & & & 0.758 \\
\hline Absence & $5(33)$ & $12(39)$ & \\
\hline Presence & $10(67)$ & $19(61)$ & \\
\hline Serum albumin, g/dL & & & 0.752 \\
\hline$\geq 3.5$ & $7(47)$ & $12(39)$ & \\
\hline$<3.5$ & $8(53)$ & $19(61)$ & \\
\hline IPI & & & 0.551 \\
\hline L/LI & 0 & $2(6)$ & \\
\hline $\mathrm{HI} / \mathrm{H}$ & $15(100)$ & $29(94)$ & \\
\hline NCCN-IPI & & & 0.551 \\
\hline L/LI & 0 & $2(6)$ & \\
\hline $\mathrm{HI} / \mathrm{H}$ & $15(100)$ & $29(94)$ & \\
\hline CNS-IPI & & & 0.040 \\
\hline Low & 0 & 0 & \\
\hline Intermediate & o & $8(26)$ & \\
\hline High & $15(100)$ & $23(74)$ & \\
\hline Hemophagocytic lymphohistiocytosis & & & 0.520 \\
\hline Absence & $11(73)$ & $19(61)$ & \\
\hline Presence & $4(26.7)$ & $12(39)$ & \\
\hline
\end{tabular}

Values are presented as number (\%).

CNS, central nervous system; ECOG, Eastern Cooperative Oncology Group; LDH, lactate dehydrogenase; IPI, International Prognostic Index; L/LI, low/low-intermediate; HI/H, high-intermediate/high; NCCN, National Comprehensive Cancer Network.

${ }^{\mathrm{a}}$ Hemoglobin $<11 \mathrm{~g} / \mathrm{dL}$, platelet count $<100 \times 10^{9} / \mathrm{L}$. 

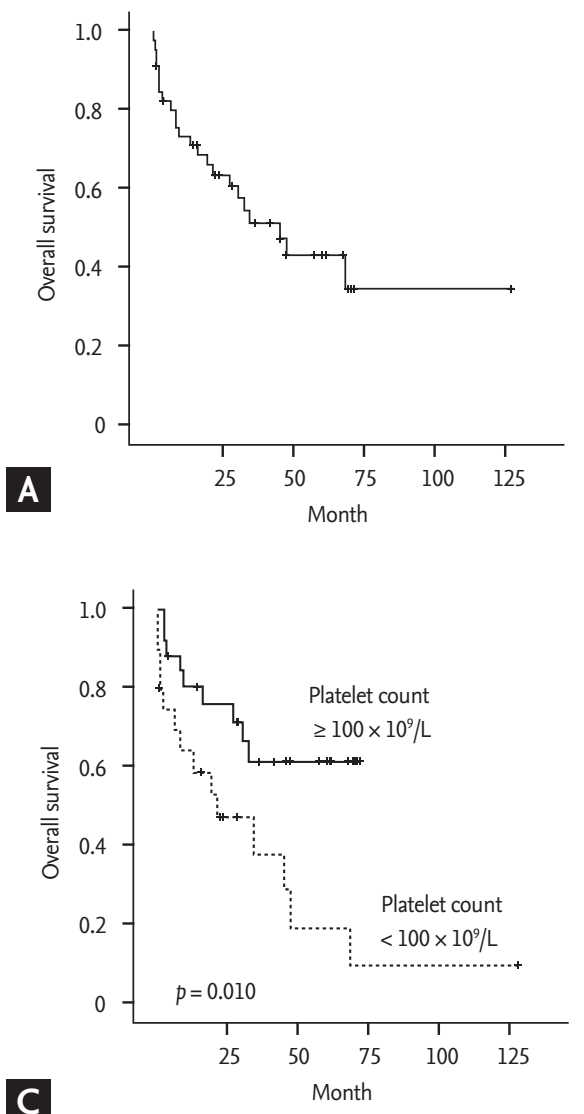
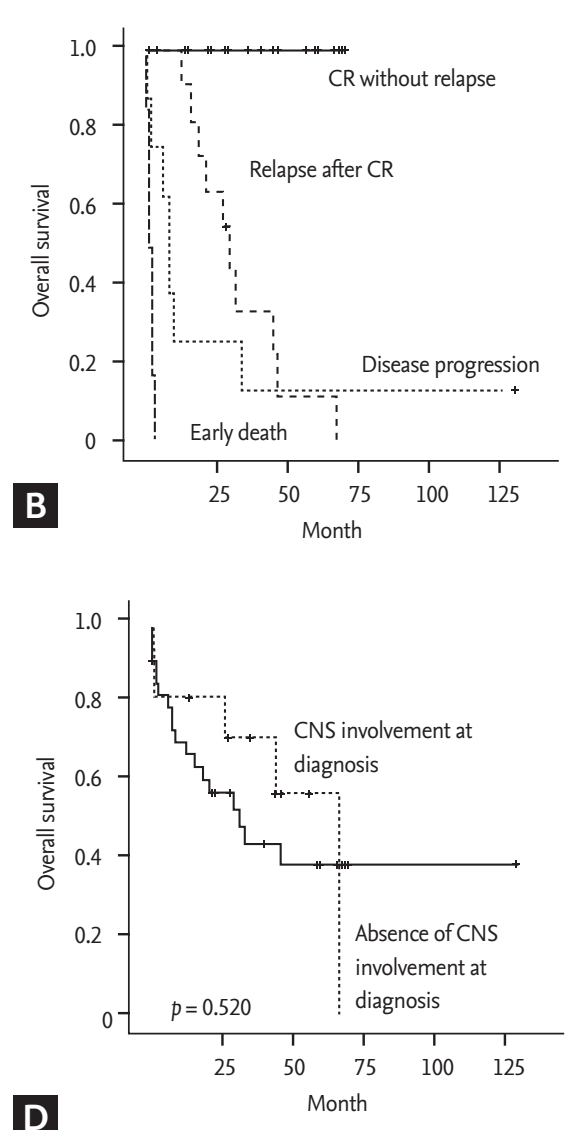

Figure 4. (A) Overall survival curves of the 46 patients. (B) The overall survival is significantly worse in patients with relapse or disease progression. (C) The overall survival is significantly associated with the presence of thrombocytopenia. (D) The overall survival is not different between patients with and without central nervous system (CNS) involvement at diagnosis. CR, complete response.

Thus, the high prevalence of MYDS8 ${ }^{L 256 P}$ and $C D 79 b$ mutations might also be associated with tumor aggressiveness and the risk of treatment failure in patients with IVLBCL.

The addition of rituximab to chemotherapy was associated with an improved CR rate (90\% vs. 50\%, p = 0.04) and 3-year OS (89\% vs. 38\%, $p=0.01$ ) compared with chemotherapy without it in Western patients [18]. A Japanese study also demonstrated a better CR rate (82\%) for patients given rituximab-containing chemotherapy rather than chemotherapy without rituximab (51\%) [19]. In our study, the median OS (45.0 months) (Fig. 4A) was similar to our previous case series reporting a median survival of 38.9 months in patients with an intravascular lymphoma treated with R-CHOP [20]. However, our CR rate $(67 \%, 31 / 46)$ was inferior to that of previous studies because eight patients showed disease progression immediately after the completion of R-CHOP even though they achieved $\mathrm{CR}$ at their interim response evaluation (Fig. 2). Furthermore, six patients died after the first or second cycle of R-CHOP $(6 / 46,13 \%)$, and the causes of death were treatment-related adverse event including cytopenia, infectious complications, and pulmonary hemorrhage. These complications might be associated with the severe toxic effects of rituximab. Actually, acute pulmonary failure after rituximab administration requiring rituximab interruption has been reported [19]. Thus, delayed administration of rituximab in the first cycle of R-CHOP should be offered to patients with a high tumor burden to prevent the occurrence of severe infusion reaction in clinical practice.

We found here that $11 / 46$ patients had CNS involve- 
ment at diagnosis (24\%) by lumbar puncture of CSF or brain MRI. In addition, four patients developed CNS relapse during follow-up. Therefore, a total of 15 patients had CNS involvement with IVLBCL (33\%, 15/46). However, the comparison of clinical features between patients with and without CNS involvement did not show significant differences (Table 2). Furthermore, all patients with CNS involvement belonged to a high-risk group according to CNS-IPI, whereas $74 \%$ of patients without CNS involvement were also grouped as highrisk by CNS-IPI, although this was only marginally statistically significant $(p=0.04)$ (Table 2 ). Thus, the risk stratification of CNS involvement as well as the prediction of CNS involvement based on the CNS-IPI score might not serve to identify patients at high risk of CNS involvement among patients with IVLBCL. Therefore, it might be difficult to establish a risk-adapted approach for the application of CNS prophylaxis in patients with the Asian variant of IVLBCL. Given the higher frequency of CNS involvement at diagnosis as well as CNS relapse in our study, a thorough evaluation of CNS including CSF analysis and brain MRI scans as well as active application of CNS prophylaxis should be considered as a routine practice for patients who are diagnosed with IVLBCL.

In our study, five patients survived out of 11 patients with CNS involvement at diagnosis after they were treated with R-CHOP plus CNS-directed therapy including high-dose MTX. As a result, the OS of patients with CNS involvement at diagnosis was not inferior to that of patients without CNS involvement at diagnosis (Fig. 4D). However, more effective treatment strategies should be developed to improve the outcome of patients with CNS involvement at diagnosis. On the other hand, the outcome of four patients who had CNS relapse after R-CHOP chemotherapy was extremely poor as three patients died from disease progression. This was consistent with the poor survival outcomes of patients with disease progression or relapse after R-CHOP (Fig. $\left.{ }_{4} \mathrm{~B}\right)$. This result implies that the prognosis of patients might be extremely poor once disease has relapsed or progressed after the first-line treatment with R-CHOP regardless of salvage chemotherapy and ASCT. This suggests the need for more effective consolidation treatments for patients at high risk of treatment failure.

A registry-based study from the European Society for Blood and Marrow Transplantation (EBMT) suggested the favorable outcome of upfront ASCT in 11 patients with IVLBCL [21]. Our study also showed better outcomes for upfront ASCT than for salvage ASCT because two patients receiving upfront ASCT including one patient with CNS involvement were still alive without re-

Table 3. Univariate and multivariate analysis for overall survival

\begin{tabular}{|c|c|c|c|c|c|c|}
\hline \multirow{2}{*}{ Variable } & \multicolumn{3}{|c|}{ Univariate analysis } & \multicolumn{3}{|c|}{ Multivariate analysis } \\
\hline & HR & $95 \%$ CI & $p$ value & HR & $95 \%$ CI & $p$ value \\
\hline Male sex & 0.733 & $0.323-1.664$ & 0.457 & 1.064 & $0.366-3.096$ & 0.910 \\
\hline Age $>60$ yr & 1.389 & $0.600-3.217$ & 0.443 & 1.156 & $0.440-3.034$ & 0.768 \\
\hline $\mathrm{ECOG} \geq 2$ & 4.092 & $0.957-17.500$ & 0.057 & 5.902 & $0.899-38.741$ & 0.064 \\
\hline B symptoms & 1.436 & $0.586-3.520$ & 0.429 & 0.611 & $0.189-1.978$ & 0.411 \\
\hline Serum LDH elevation $(>3)$ & 1.322 & $0.542-3.224$ & 0.539 & 0.404 & $0.122-1.334$ & 0.137 \\
\hline Hemoglobin < $11 \mathrm{~g} / \mathrm{dL}$ & 2.587 & $1.014-6.597$ & 0.047 & 2.182 & $0.578-8.235$ & 0.250 \\
\hline Platelet count $<100 \times 10^{9} / \mathrm{L}$ & 2.865 & $1.233-6.652$ & 0.014 & 1.641 & $0.497-5.425$ & 0.417 \\
\hline Hepatosplenomegaly & 2.313 & $0.946-5.656$ & 0.066 & 1.182 & $0.378-3.702$ & 0.774 \\
\hline Bone marrow involvement & 1.849 & $0.779-4.390$ & 0.164 & 1.025 & $0.345-3.045$ & 0.964 \\
\hline High-risk of CNS-IPI & 2.705 & $0.629-11.628$ & 0.181 & 1.783 & $0.286-11.115$ & 0.536 \\
\hline Presence of HLH & 1.157 & $0.468-2.863$ & $0.75^{2}$ & 1.061 & $0.348-3.235$ & 0.917 \\
\hline CNS involvement $^{\mathrm{a}}$ & 0.723 & $0.267-1.957$ & 0.723 & 0.508 & $0.131-1.980$ & 0.329 \\
\hline
\end{tabular}

HR, hazard ratio; CI, confidence interval; ECOG, Eastern Cooperative Oncology Group; LDH, lactate dehydrogenase; CNS, central nervous system; IPI, International Prognostic Index; HLH, hemophagocytic lymphohistiocytosis.

${ }^{\mathrm{a}} \mathrm{CNS}$ involvement at diagnosis $(\mathrm{n}=11)$. 
lapse at the end of the study period (Fig. 3). However, the numbers were too small in our study and that of the EBMT. Given that more than $60 \%$ of patients with $\mathrm{CR}$ after R-CHOP maintained their response without upfront ASCT, it might be important to select patients who are candidates for upfront ASCT rather than applying ASCT to all patients with IVLBCL.

In our study, only thrombocytopenia was significantly associated with a poor OS (Fig. 4C). However, it failed to show an independent association with poor OS in the multivariate analysis (Table 3). Other parameters that were believed to be unfavorable, such as the presence of HLH, did not show prognostic relevance. As this lack of significant risk factors for poor survival outcomes might be associated with the relatively few patients in our study as well as the retrospective nature of the study, a well-designed prospective study with a larger study population is warranted to explore prognostic factors for IVLBCL and the role of upfront ASCT in the future.

\section{KEY MESSAGE}

1. The median overall survival of patients with Asian variant of intravascular large B-cell lymphoma was 45.0 months, and overall survival was not different according to the involvement of central nervous system (CNS).

2. The prediction of CNS involvement based on the clinical features including CNS-International Prognostic Index might not serve to identify patients at high risk of CNS involvement.

\section{Conflict of interest}

No potential conflict of interest relevant to this article was reported.

\section{REFERENCES}

1. Murase T, Nakamura S, Kawauchi K, et al. An Asian variant of intravascular large B-cell lymphoma: clinical, pathological and cytogenetic approaches to diffuse large B-cell lymphoma associated with haemophagocytic syndrome. Br J Haematol 2000;111:826-834.
2. Swerdlow SH, Campo E, Pileri SA, et al. The 2016 revision of the World Health Organization classification of lymphoid neoplasms. Blood 2016;127:2375-2390.

3. Murase T, Yamaguchi M, Suzuki R, et al. Intravascular large B-cell lymphoma (IVLBCL): a clinicopathologic study of 96 cases with special reference to the immunophenotypic heterogeneity of CD5. Blood 2007;109:478-485.

4. Ponzoni M, Ferreri AJ, Campo E, et al. Definition, diagnosis, and management of intravascular large B-cell lymphoma: proposals and perspectives from an international consensus meeting. J Clin Oncol 2007;25:3168-3173.

5. Ferreri AJ, Campo E, Seymour JF, et al. Intravascular lymphoma: clinical presentation, natural history, management and prognostic factors in a series of 38 cases, with special emphasis on the 'cutaneous variant'. Br J Haematol 2004;127:173-183.

6. Ferreri AJ, Dognini GP, Campo E, et al. Variations in clinical presentation, frequency of hemophagocytosis and clinical behavior of intravascular lymphoma diagnosed in different geographical regions. Haematologica 2007;92:486-492.

7. Rajyaguru DJ, Bhaskar C, Borgert AJ, Smith A, Parsons B. Intravascular large B-cell lymphoma in the United States (US): a population-based study using Surveillance, Epidemiology, and End Results program and National Cancer Database. Leuk Lymphoma 2017;58:1-9.

8. Feugier P, Virion JM, Tilly H, et al. Incidence and risk factors for central nervous system occurrence in elderly patients with diffuse large-B-cell lymphoma: influence of rituximab. Ann Oncol 2004;15:129-133.

9. Bjorkholm M, Hagberg H, Holte $\mathrm{H}$, et al. Central nervous system occurrence in elderly patients with aggressive lymphoma and a long-term follow-up. Ann Oncol 2007;18:1085-1089.

10. Boehme V, Schmitz N, Zeynalova S, Loeffler M, Pfreundschuh M. CNS events in elderly patients with aggressive lymphoma treated with modern chemotherapy (CHOP14) with or without rituximab: an analysis of patients treated in the RICOVER-6o trial of the German HighGrade Non-Hodgkin Lymphoma Study Group (DSHNHL). Blood 2009;113:3896-3902.

11. Kim SJ, Oh SY, Kim JS, et al. Secondary central nervous system (CNS) involvement in patients with diffuse large B-cell lymphoma: a therapeutic dilemma. Ann Hematol 2011;90:539-546.

12. Schmitz N, Zeynalova S, Nickelsen M, et al. CNS Interna- 
tional Prognostic Index: a risk model for CNS relapse in patients with diffuse large B-cell lymphoma treated with R-CHOP. J Clin Oncol 2016;34:3150-3156.

13. Zhou Z, Sehn LH, Rademaker AW, et al. An enhanced International Prognostic Index (NCCN-IPI) for patients with diffuse large B-cell lymphoma treated in the rituximab era. Blood 2014;123:837-842.

14. Cheson BD, Pfistner B, Juweid ME, et al. Revised response criteria for malignant lymphoma. J Clin Oncol 2007;25:579-586.

15. Su DW, Pasch W, Costales C, Siddiqi I, Mohrbacher A. Asian-variant intravascular large B-cell lymphoma. Proc (Bayl Univ Med Cent) 2017;30:186-189.

16. Schrader AMR, Jansen PM, Willemze R, et al. High prevalence of MYD88 and CD79B mutations in intravascular large B-cell lymphoma. Blood 2018;131:2086-2089.

17. Schmitz R, Wright GW, Huang DW, et al. Genetics and pathogenesis of diffuse large B-Cell lymphoma. N Engl J Med 2018;378:1396-1407.
18. Ferreri AJ, Dognini GP, Bairey O, et al. The addition of rituximab to anthracycline-based chemotherapy significantly improves outcome in 'Western' patients with intravascular large B-cell lymphoma. Br J Haematol 2008;143:253-257.

19. Shimada K, Matsue K, Yamamoto K, et al. Retrospective analysis of intravascular large B-cell lymphoma treated with rituximab-containing chemotherapy as reported by the IVL study group in Japan. J Clin Oncol 2008;26:31893195 .

20. Hong JY, Kim HJ, Ko YH, et al. Clinical features and treatment outcomes of intravascular large B-cell lymphoma: a single-center experience in Korea. Acta Haematol 2014;131:18-27.

21. Meissner J, Finel H, Dietrich S, et al. Autologous hematopoietic stem cell transplantation for intravascular large B-cell lymphoma: the European Society for Blood and Marrow Transplantation experience. Bone Marrow Transplant 2017;52:650-652. 